\title{
Dynamical Chaos and Lateral Transport of a Passive Scalar in the Annular Reverse Jet Flow
}

\author{
V.P. Reutov，G. V.Rybushkina
}

The transition to dynamical chaos and the related lateral (cross-flow) transport of a passive scalar in the reverse annular jet flow generating two chains of wave-vortex structures are studied. The quasi-geostrophic equations for the barotropic (quasi-two-dimensional) flow written in polar coordinates with allowance for the beta-effect and external friction are solved numerically using a pseudospectral method. The critical parameters of the equilibrium flow with a complex "two-hump" azimuth velocity profile facilitating a faster transition to the complex dynamics are determined. Two regular multiharmonic regimes of wave generation are revealed with increasing flow supercriticality before the onset of Eulerian chaos. The occurrence of the complex flow dynamics is confirmed by a direct calculation of the largest Lyapunov exponent. The evolution of streamline images is analyzed by making video, thereby chains with single and composite structures are distinguished. The wavenumber-frequency spectra confirming the possibility of chaotic transport of the passive scalar are drawn for the basic regimes of wave generation. The power law exponents for the azimuth particle displacement and their variance, which proved the occurrence of the anomalous azimuth transport of the passive scalar, are determined. Lagrangian chaos is studied by computing the finite-time Lyapunov exponent and its distribution function. The internal chain (with respect to the annulus center) is found to be totally subject to Lagrangian chaos, while only the external chain boundary is chaotic. It is revealed that the cross-flow transport occurs only in the regime of Eulerian dynamical chaos, since there exists a barrier to it in the multiharmonic regimes. The images of fluid particles confirming the presence of lateral transport are obtained and their quantitative characteristics are determined.

Keywords: barotropic jet flow, chains of wave structures, Eulerian and Lagrangian chaos, cross-flow chaotic transport

Received July 6, 2021

Accepted July 26, 2021

This work was supported by the Ministry of Science and Higher Education of the Russian Federation within the State task for the Institute of Applied Physics of RAS (project No. 0030-2021-0011).

Vladimir P. Reutov

reutov@appl.sci-nnov.ru

Galina V. Rybushkina

ryb@appl.sci-nnov.ru

Institute of Applied Physics, RAS

ul. Ulyanova 46, Nizhny Novgorod, 603950 Russia

RUSSIAN JOURNAL OF NONLINEAR DYNAMICS, 2021, 17(3), 263-274 


\section{Introduction}

The barotropic instability of zonal flows in the atmosphere and oceans of the Earth and other planets as well as in the related experiments gives rise to the generation of chains of wave structures with closed streamlines, which may essentially influence the chaotic transport of passive scalars (e.g., $[4,5,9,13,17])$. The investigation of the chaotic transport in jet zonal flows with a reverse velocity profile is of great interest in connection with the phenomenon of atmospheric blocking responsible for long-lived weather anomalies [3, 7]. In our previous work [11] we were concerned with the transition to Eulerian dynamical chaos and associated anomalous transport in a reverse barotropic jet described within the framework of the plane-parallel flow model. A complex velocity profile facilitating a faster transition to chaos was considered. The main attention was focused on immovable chains arising at a certain degree of flow reversibility. In this paper we study the azimuth and lateral (cross-flow) transport related to Eulerian and Lagrangian chaos in an annular barotropic jet with a reverse profile of azimuth velocity.

Currently there are a lot of studies concerning the cross-flow transport and chaotic mixing in the Gulf Stream and other oceanic meandering jets $[9,13,17]$. In the first works, the Hamiltonian description of motion of the fluid particles was elaborated based on the kinematic flow model and the notions of stable and unstable manifolds $[2,15]$. A brief description of this approach and a large number of citations of the literature relevant to the question were given in [13] together with the dynamically consistent flow model confirming the results of the kinematic theory. Later investigations of the cross-flow transport in the Gulf Stream were presented in the overview [9].

The phenomenon of the lateral wave transport that results in spreading of passive scalars (materials and others) across the flow is of interest for a wide range of barotropic and baroclinic flows producing chains of structures with closed streamlines. The processes of chaotic advection of the passive scalars from the vortex core to a free stream have been widely studied for the flows containing point and other vortices (e.g., $[1,8,14,17])$. This work is concerned with the investigation of the anomalous and cross-flow wave transport in the annular reverse jet with a complex "two-hump" azimuth velocity profile. The main attention is focused on the generation of slow chains of structures with closed streamlines described within a dynamically consistent flow model. The walls of the annular channel are supposed to be rigid and nonpercolating. The quasi-geostrophic equations for a barotropic (quasi-two-dimensional) flow with allowance for the beta-effect and external friction written in polar coordinates are solved by the pseudospectral method borrowed from the work [12]. To study the transport phenomena, the equations for the tracer particles are introduced in the numerical scheme. The transition to Eulerian chaos with increasing flow velocity is considered and the relationship between Eulerian and Lagrangian chaos producing chaotic cross-flow transport is elucidated.

\section{Basic equations}

Let us consider a quasi-two-dimensional (barotropic) annular flow using the beta-plane approximation and taking external (bottom) friction into account. The dimensionless variables are introduced using a maximal flow velocity $U$ and a radial velocity shift $L$. The annular channel is supposed to have a midradius $r_{0}$ and wall coordinates $r=r_{0} \pm b$, where $b$ is the channel halfwidth. The basic equations will be written in the polar coordinates $(r, \theta)$ in terms of absolute 
vorticity $\zeta$ and streamfunction (see, e.g., [16]):

$$
\frac{\partial \zeta}{\partial t}+\frac{1}{r}\left(\frac{\partial \psi}{\partial \theta} \frac{\partial \zeta}{\partial r}-\frac{\partial \psi}{\partial r} \frac{\partial \zeta}{\partial \theta}\right)-\frac{\beta}{r} \frac{\partial \psi}{\partial \theta}=-\lambda\left(\zeta-\zeta_{0}\right)+\mathrm{R}^{-1} \Delta\left(\zeta-\zeta_{0}\right), \quad \Delta \psi=-\zeta,
$$

where $\Delta=\partial^{2} / \partial r^{2}+(1 / r) \partial / \partial r+\left(1 / r^{2}\right) \partial^{2} / \theta^{2}$ is the Laplacian, $\mathrm{R}=U L / \nu$ is the Reynolds number ( $\nu$ is the effective kinematic viscosity of the fluid), $\lambda=\lambda^{* *} L / U$ and $\beta=\beta^{* *} L^{2} / U$ are the dimensionless analogs of the external friction coefficient $\lambda^{* *}$ and the Coriolis parameter gradient $\beta^{* *}$, respectively; $\zeta_{0}=\left(\partial v_{0}(r) / \partial r\right)+(1 / r) v_{0}(r)$ is the equilibrium vorticity, and $v_{0}(r)$ is the equilibrium azimuth velocity profile. The terms $\sim \zeta_{0}$ in (2.1) simulate an external agency supporting the equilibrium flow with velocity profile $v_{0}(r)$. Correspondingly, the radial and azimuth velocity components are related to the streamfunction by the expressions

$$
v_{r}=\frac{1}{r} \frac{\partial \psi}{\partial \theta}, \quad v_{\theta}=-\frac{\partial \psi}{\partial r} .
$$

All variables are represented as a superposition of mean and oscillating (with period $2 \pi$ in $\theta$ ) components:

$$
\zeta=\bar{\zeta}(r, t)+\widetilde{\zeta}, \quad \psi=-\int \bar{v}_{\theta}(r, t) d r+\widetilde{\psi}, \quad v_{\theta}=\bar{v}_{\theta}(r, t)-\frac{\partial \widetilde{\psi}}{\partial r},
$$

where $\bar{\zeta}=\left(\partial \bar{v}_{\theta}(r, t) / \partial r\right)+(1 / r) \bar{v}_{\theta}(r, t)$ and $\bar{v}_{\theta}(r, t)$ are the mean vorticity and mean azimuth velocity of the annular flow, while the oscillating variables are marked by a tilde. The boundary conditions $\partial \widetilde{\psi} / \partial \theta=0, \partial \widetilde{\theta} / \partial r=0$, and $\bar{v}_{\theta}=0$ are specified at the channel walls $r=r_{0} \pm b$. The oscillating variables are sought in the form of the truncated Fourier series

$$
(\widetilde{\psi}, \widetilde{\zeta})=\sum_{\substack{m=-K \\ m \neq 0}}^{K}\left(\widehat{\psi}_{m}(r, t), \widehat{\zeta}_{m}(r, t)\right) e^{i m \theta}
$$

where $K$ is the number of "nonzero" harmonics, $\widehat{\zeta}_{m}$ and $\widehat{\psi}_{m}$ are the complex profiles of the $m$ th harmonic of vorticity and streamfunction, respectively. The simultaneous system of equations governing the profiles $\widehat{\psi}_{m}, \widehat{\zeta}_{m}$ and mean velocity $\bar{v}_{\theta}$ including the boundary conditions were derived in [12].

We utilize the velocity profile $v_{0}(y)$ of the equilibrium reverse jet flow specified by formulae (2.4) of [11] replacing the Cartesian coordinate $y$ by $r$. The numerical coefficients of the profile approximation are also taken the same as in [11]. In this case, the conditions $v_{0}(r \pm b)=0$ are met to a high accuracy and the maximal value of $v_{0}$ across the annular channel is equal to unity regardless of the magnitude of the reverse parameter, allowing the contribution of the reverse flow in the entire jet drain to be controlled. By varying the reverse parameter (designated now by $q$ to exclude the coincidence with the notation $b$ adopted above) the wave structures with zero or very small velocities were obtained in the jet. The main computations were performed for the annulus parameters $r_{0}=6.5$ and $b=4$, and the equilibrium velocity profile $v_{0}(r)$ obtained at $q=0.758$ is shown in Fig. 1 by curve 1 .

\section{The transition to chaos}

To study the nonlinear regimes of chain generation, the solution of the linear eigenvalue problem was first sought numerically for the perturbations $\sim \exp (i m \theta-i \omega t)$, where $m=$ 


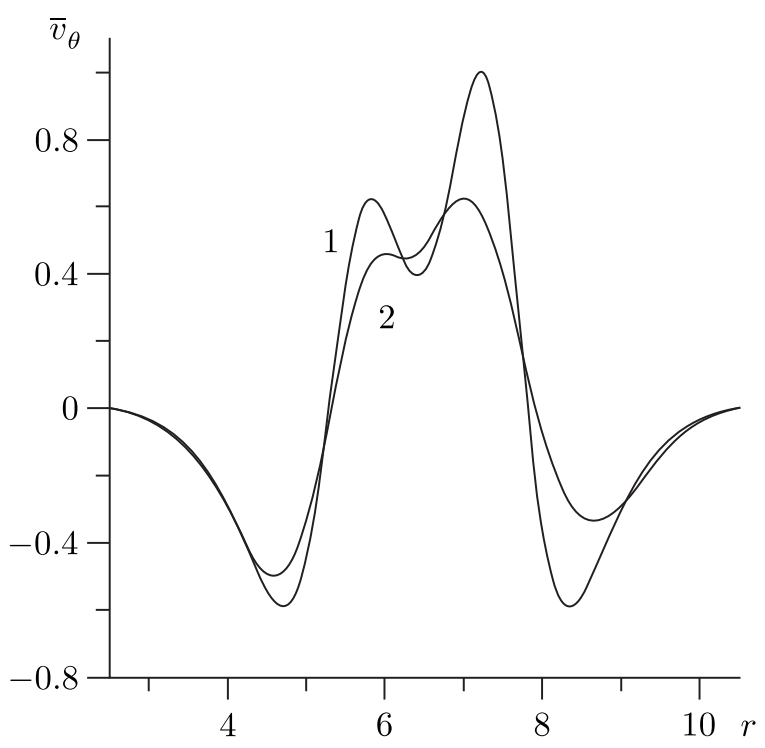

Fig. 1. The azimuth velocity profiles of the annular reverse jet between the channel walls $2.5 \leqslant r \leqslant 10.5$ : curve 1 corresponds to the equilibrium velocity profile $v_{0}(r)$, while curve 2 shows the mean velocity profile $\bar{v}_{\theta}$ at $\beta_{c}=0.8, \mathrm{R}_{c}=120, \gamma=3.108$. In both cases $q=0.758$.

$=1,2, \ldots$ is the wave number and $\omega$ is the cyclic frequency. We introduce the quantity $\Omega=$ $=\operatorname{Re} \omega / m$ that is an angular phase velocity of the wave with wavenumber $m$ coinciding with the angular frequency of rotation of the chain produced by this wave. The solution of the eigenvalue problem was discussed in detail in $[10,11]$ for the plane-parallel flows and in [12] for the annular flow. Following $[10,12]$, we introduce the critical velocity $U_{c}$, at which the instability occurs first, and the associated supercriticality $\gamma=U / U_{c}$. The dimensionless parameters corresponding to the instability threshold are designated by $\mathrm{R}_{c}, \beta_{c}$, and $\lambda_{c}$, while the critical wavenumber and angular velocity are $m_{c}$ and $\Omega_{c}$, respectively [12]. An increase in $\gamma$ at constant $\lambda^{* *}, \beta^{* *}$, and $\nu$ leads to the variation of $\mathrm{R}, \beta$, and $\lambda$ in accordance with the relations $\gamma=\mathrm{R} / \mathrm{R}_{c}=\lambda_{c} / \lambda=$ $=\beta_{c} / \beta$. The critical parameters $\lambda_{c}, m_{c}$, and $\Omega_{c}$ found for the first two flow modes for the preset values $\mathrm{R}=\mathrm{R}_{c}$ and $\beta=\beta_{c}$ are shown in Table 1 (recall that $\lambda_{c}$ coincides with the maximal instability increment $\operatorname{Im} \omega(m)$ at $\lambda=0[5,10,12])$. Values of the reverse parameters are chosen to achieve the angular phase velocity of the main mode 1 at the critical point very close to zero at $\beta=0$ and at $\beta=0.8$. Table 1 shows that the instability threshold is shifted significantly at passage to $\beta=0.8$ for both modes.

Table 1. Determination of the critical parameters $\lambda_{c}, m_{c}$, and $\Omega_{c}$ of two most unstable modes (numbered 1 and 2) at different reversibility $q$.

\begin{tabular}{cccccccc}
\hline$q$ & Mode & $\mathrm{R}_{c}$ & $\beta_{c}$ & $\lambda_{c}$ & $m_{c}$ & $\Omega_{c}$ \\
\hline 0.824 & 1 & 120 & 0 & 0.5290 & 12 & $3.3 \cdot 10^{-6}$ \\
0.824 & 2 & 120 & 0 & 0.3913 & 7 & -0.0356 \\
0.758 & 1 & 120 & 0.8 & 0.3686 & 12 & $8.7 \cdot 10^{-5}$ \\
0.758 & 2 & 120 & 0.8 & 0.2720 & 8 & -0.0372 \\
\hline
\end{tabular}

To study the nonlinear development of barotropic instability in the annular reverse jet flow, the equations for the complex amplitude profiles $\widehat{\zeta}_{m}, \widehat{\psi}_{m}$, and the mean azimuth velocity $\bar{v}_{\theta}$ were 
solved numerically using the scheme described in [12]. The complex discrete Fourier transform with respect to the $\theta$ coordinate, including $N=128$ spatial harmonics, was processed using the algorithm of Fast Fourier Transform (FFT). To exclude the aliasing phenomena we took $K=42$. Note that in this case the third harmonic $m=36$ of the highest mode $m=12$ which must be small for the vorticity field is taken into account. Discretization along the $r$ coordinate with a constant step containing 240 grid points was made. The main computations were performed for the annular channel with the aforementioned parameters. The noisy initial conditions for the amplitudes and phases of the harmonics were specified (see [12] for details). To study properties of the scalar transport, we solved the simultaneous equations for the annular flow and the tracer particles.

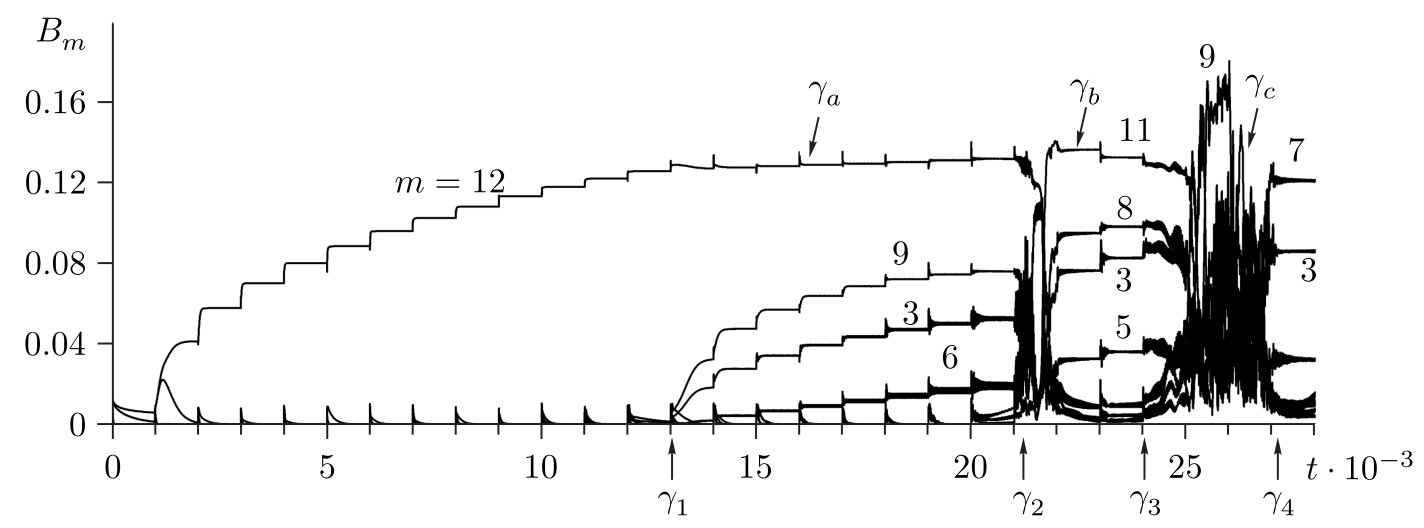

Fig. 2. Temporal evolution of the integral amplitudes of harmonics of the azimuth velocity with supercriticality increasing step by step (starting from $\gamma=1$ ) over the time interval $\Delta t=1000$. Values of $m$ show the wavenumbers of the predominant harmonics, while supercriticalities $\gamma_{1}=1.545, \gamma_{2}=2.324$, $\gamma_{3}=2.866$, and $\gamma_{4}=3.738$ correspond to passages between different wave generation regimes, while $\gamma_{a}=1,767, \gamma_{b}=2.480$, and $\gamma_{c}=3.108$ are the reference points. For this and the next figures $\beta_{c}=0.8$, $\mathrm{R}_{c}=120$.

We suppose that the leading parameter is the supercriticality $\gamma$, while the other dimensionless parameters follow the changes in $\gamma$ at constant at constant $\lambda^{* *}, \beta^{* *}$, and $\nu$. Taking into account the relation $\gamma=\lambda_{c} / \lambda$, we altered $\gamma$ by decreasing $\lambda$ step by step from $\lambda=\lambda_{c}$ with a small decrement $\Delta \lambda=0.01$. In accordance with Table 1 , we took $\lambda_{c}=0.369, \mathrm{R}_{c}=120$, and $\beta_{c}=0.8$. After each jump, the parameter $\lambda$, as well as the related quantities $\gamma, \mathrm{R}$, and $\beta$, were maintained constant during the time interval $\Delta t=1000$ up to the next step (see $[10,12]$ for details). Figure 2 shows the evolution diagram mapping the behavior of the integral amplitudes of spatial harmonics of the azimuth velocity $B_{m}=\left(\int_{r_{0}-b}^{r_{0}+b}\left|\widehat{v}_{\theta m}\right|^{2} d r\right)^{1 / 2}$, where $m$ is the number of the harmonic and $\widehat{v}_{\theta m}=-\partial \widehat{\psi}_{m}(r, t) / \partial r$. The supercriticalities $\gamma_{j}(j=1-4)$ separating different regimes of generation are given in the figure caption. The reference points used for the analysis of these regimes are marked by $\gamma_{a}, \gamma_{b}$ and $\gamma_{c}$. Figure 2 shows that a single-mode regime with basic harmonics $m=12$ arises first at $\gamma>1$, which is consistent with Table 1 . Thereafter two multiharmonic regimes appear sequentially at $\gamma_{1}$ and $\gamma_{2}$ until the chaotic generation is established at $\gamma_{3}<\gamma<\gamma_{4}$. The appearance of harmonics with lower wavenumbers in the multiharmonic regimes may be explained by the excitation of the second mode given in Table 1 (some distinctions are possible due to deformation of the mean velocity profile similar to the one in Fig. 1). Besides, the wavenumbers of the harmonics produced by the "nonlinear subtraction" 


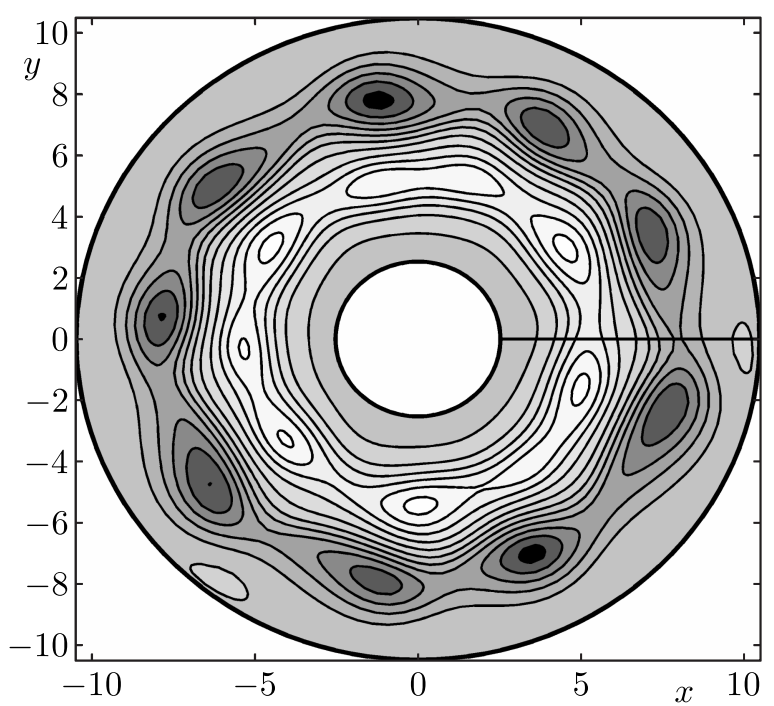

(a)

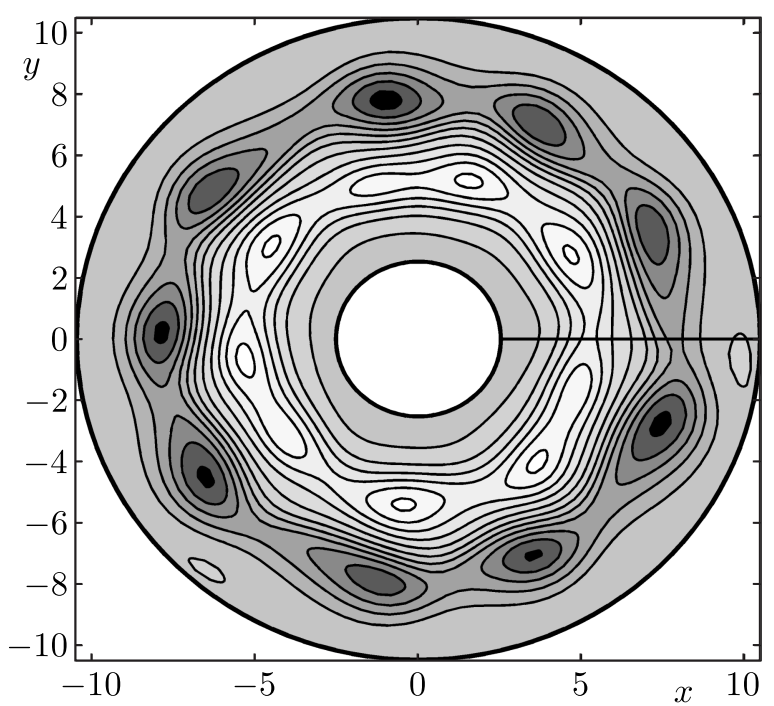

(b)

Fig. 3. Sequential snapshots of streamlines of the annular jet flow obtained for $\gamma=\gamma_{c}$ in the reference frame corotating with the angular frequency of the external chain $\Omega=0.0016$. The time interval between the images (a) and (b) is equal to 10 (the straight lines mark the angle $\theta=0 ; x$ and $y$ are the Cartesian coordinates).

can be seen in Fig. 2. The existence of Eulerian chaos was proved by direct computation of the largest Lyapunov exponent, which turned out to be equal to 0.01 at $\gamma=\gamma_{c}$ (see [10] for details). Note that the next appearance of the chaotic regime was revealed at a higher supercriticality $(\gamma>5.373)$.

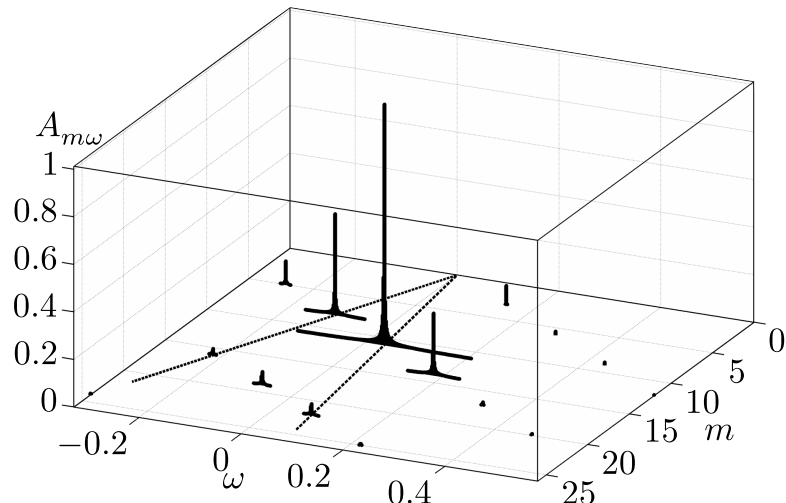

(a)

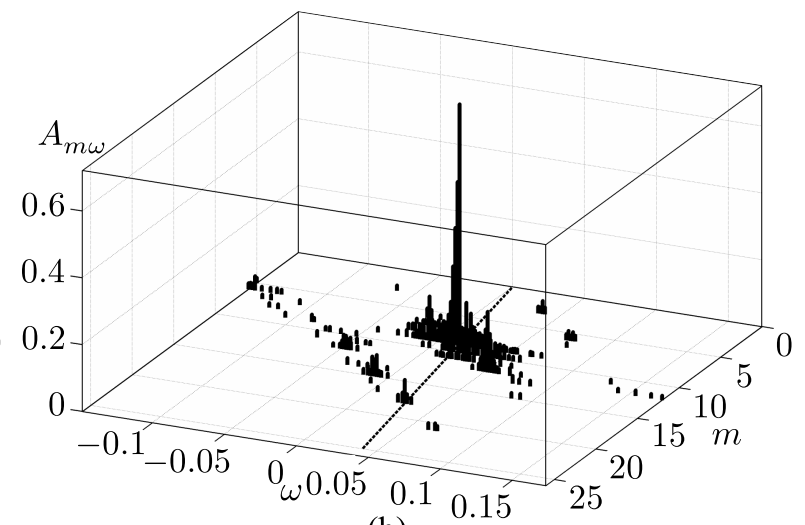

(b)

Fig. 4. Wavenumber-frequency spectra of azimuth velocity at the inside boundary of the external chain $(r=7.16)$ in the laboratory reference frame: (a) $\gamma=\gamma_{b}$, the straight lines correspond to $\Omega=0.0033$ and $\Omega=-0.0136$ for the first and second largest peaks, respectively; (b) $\gamma=\gamma_{c}$, the straight line corresponds to $\Omega=0.0016$.

The behavior of the streamlines is studied by making videos. The sequence of images of streamlines in the chaotic regime, drawn in a corotating reference frame of an external chain (localized near the external boundary of the annulus), is displayed in Fig. 3. Comparison of Figs. 3a and 3b shows that the external chain contains 9 immovable "single" structures with closed 
streamlines surrounded by a single separatrix contour. The angular frequency of chain rotation in the laboratory reference frame remains rather small $(\Omega=0.0016)$ and can be made exactly zero by a small variation of $q$, as was done in [10]. The internal chain also contains 9 immovable single structures, which indicates synchronization of the chain rotations. Besides, the double and triple composite structures including two and three single structures surrounded by common separatrix contours can be seen in the internal chain. The composite structures rapidly mutate by reconnection of the closed streamlines, but the boundaries of the closed streamlines and, therefore, the rotation of the comoving reference frame are determined by the single structures, i. e., their angular frequency is also equal to $\Omega=0.0016$. At all reference points, including $\gamma_{c}$, the streamlines contain modulated structure chains with the modulation increasing with an increase in $\gamma$. A similar behavior of the wave structures was revealed in the Kolmogorov flow [12].

It is useful to supplement the evolution diagram in Fig. 2 by the wavenumber-frequency spectra taken at the level $r=$ const located near the inside boundary of closed streamlines of the external chain in Fig. 3. The multiharmonic regimes arising at $\gamma=\gamma_{a}$, and $\gamma=\gamma_{b}$ are characterized by discrete wavenumber-frequency spectra, one of which is presented in Fig. 6a, whereas Fig. 6b demonstrates the "continuous" spectrum arising at chaotic generation. Note that the secondary discrete peaks in Fig. 6 are responsible for the appearance of the composite structures in Fig. 3, as was noted in [12]. Both spectra in Fig. 6 show that the spectral components with different angular velocities, which is needed to form the stochastic layers along the boundaries of the closed streamlines, are generated at saturation of the flow instability.

\section{The azimuth and lateral transport}

To prove the occurrence of azimuth chaotic transport, we calculated the mean angular displacement $M=\langle\delta \theta\rangle$ and the variance $\sigma^{2}=\left\langle(\delta \theta-\langle\delta \theta\rangle)^{2}\right\rangle$ of tracer particles $\left(\delta \theta=\theta^{\prime}(t)-\theta^{\prime}(0)\right.$, the angle brackets designate ensemble averaging, and the primes the comoving reference frame of the chain considered) [12]. The initial conditions were specified along the fixed pseudoseparatrix contours determined in the comoving reference frame at $t=0$ arbitrarily taken in the established generation regime. In accordance with the definition of [10], these contours go through all saddle points and lie along the boundaries of the closed streamlines. The power laws $|M| \sim t^{s}$ and $\sigma^{2} \sim t^{\alpha}$ were obtained over the time interval $10^{2} \lesssim t \lesssim 10^{3}$ and their exponents $s$ and $\alpha$ were computed in the reference frames rotating with the angular frequency of the chains for all reference points. The results of the computations are presented in Table 2 . It can be seen that the mean displacement is normal (or close to normal) and the anomalous diffusion (superdiffusion) occurs at all reference points (see the definitions in [4]).

Table 2. Power law exponents for the internal and external chains at the reference points marked in Fig. 2.

\begin{tabular}{cccc}
\hline chain & $\gamma$ & $s$ & $\alpha$ \\
\hline int. & $\gamma_{a}$ & 0.968 & 1.531 \\
ext. & $\gamma_{a}$ & 1.00 & 1.928 \\
int. & $\gamma_{b}$ & 0.869 & 1.438 \\
ext. & $\gamma_{b}$ & 0.985 & 1.769 \\
int. & $\gamma_{c}$ & 1.00 & 1.884 \\
ext. & $\gamma_{c}$ & 1.00 & 1.938 \\
\hline
\end{tabular}


Localization of the flow regions subject to Lagrangian chaos was evaluated by calculating the finite-time Lyapunov exponents (FTLE) for the fluid particles in a single segment of the flow. To this end, we utilized the scheme presented in [6] based on the variation of particle trajectories. The equations of motion of the tracer particles in polar coordinates have the form

$$
\frac{d r}{d t}=\frac{1}{r} \frac{\partial \widetilde{\psi}}{\partial \theta}, \quad \frac{d \theta}{d t}=\frac{1}{r} \bar{v}_{\theta}(r, t)-\frac{1}{r}\left(\frac{\partial \widetilde{\psi}}{\partial r}\right) .
$$

By varying the Lagrangian orbits of the fluid particles around the reference orbit $[r(t), \theta(t)]$ defined by (4.1) we obtain the following system of equations:

$$
\begin{gathered}
\frac{d \delta r}{d t}=\left(-\frac{1}{r^{2}} \frac{\partial \widetilde{\psi}}{\partial \theta}+\frac{1}{r} \frac{\partial^{2} \widetilde{\psi}}{\partial \theta}\right) \delta r+\frac{1}{r} \frac{\partial^{2} \widetilde{\psi}}{\partial \theta^{2}} \delta \theta \\
\frac{d \delta \theta}{d t}=\left(-\frac{1}{r^{2}} \bar{v}_{\theta}(r, t)+\frac{1}{r} \frac{\partial \bar{v}_{\theta}(r, t)}{\partial r}+\frac{1}{r^{2}} \frac{\partial \widetilde{\psi}}{\partial r}-\frac{1}{r} \frac{\partial^{2} \widetilde{\psi}}{\partial r^{2}}\right) \delta r-\frac{1}{r}\left(\frac{\partial^{2} \widetilde{\psi}}{\partial r \partial \theta}\right) \delta \theta,
\end{gathered}
$$

where the FTLE value $h$ is determined by

$$
h=\frac{1}{T} \ln \left[\frac{|\delta \vec{l}(T)|}{|\delta \vec{l}(0)|}\right] \quad\left(|\delta \vec{l}|=\sqrt{(\delta r)^{2}+r^{2}(\delta \theta)^{2}}\right) .
$$

Here, $\delta \vec{l}$ is the displacement vector of the particle orbit and $T$ is the time interval. The Lyapunov exponent $h$ tends to a constant value when the time interval $T$ is large enough, but this may be violated in some flow regions. The initial time $t=0$ must be chosen within the established regime of generation.

The simultaneous system of equations (4.1)-(4.3) was solved together with the equations governing the flow fields and the FTLE was determined by (4.4) at the largest value of $T$, for which the exponents given in Table 2 were obtained authentically. The particles were initially placed in the nods of a uniform $100 \times 100$ grid covering a segment of the annulus channel in Fig. 3a from the straight line $\theta=0$ to the counterclockwise line $\theta=0.69$, which contains one cell of each chain. Figure 5a demonstrates the FTLE image in negative grayscale (black and white colors designate maximal and minimal $h$, respectively). Similar pictures were obtained in [6] for the Kelvin - Helmholtz instability using another color map. The $h$ distribution is almost uniform inside the "integrable" regions of the flow [6], while the Lagrangian chaos is localized along the current separatrix contour of the external chain and inside the separatrix contour of the internal chain, provided that these contours are drawn in the comoving reference frame of each chain (see [6] for details). Note that the chaotic region of the external chain broadens in the vicinity of the unstable manifold of the hyperbolic point $[2,13,15,17]$. The localization of chaos inside the internal chain confirms the statement that the Eulerian chaos appears when Lagrangian chaos penetrates the vortex cores [6]. Figure 5b shows the FTLE distribution function found at the grid pointed above. It allows the characteristic values of $h$ to be determined. The two-hump vertex of the distribution function may be explained by the occurrence of two different chaotic regions in Fig. 5a. The sharp peak displayed in Fig. 5b at a very small $h$ is related to the contribution of the velocity shift in the integrable regions, as was noted in [6].

For studying lateral (cross-flow) transport in the reverse jet flow, we specified the initial conditions for the tracer particles at $t=0$ along the pseudoseparatrix contours of the chains, or inside these contours. The cross-flow transport was revealed only for the chaotic regime of 


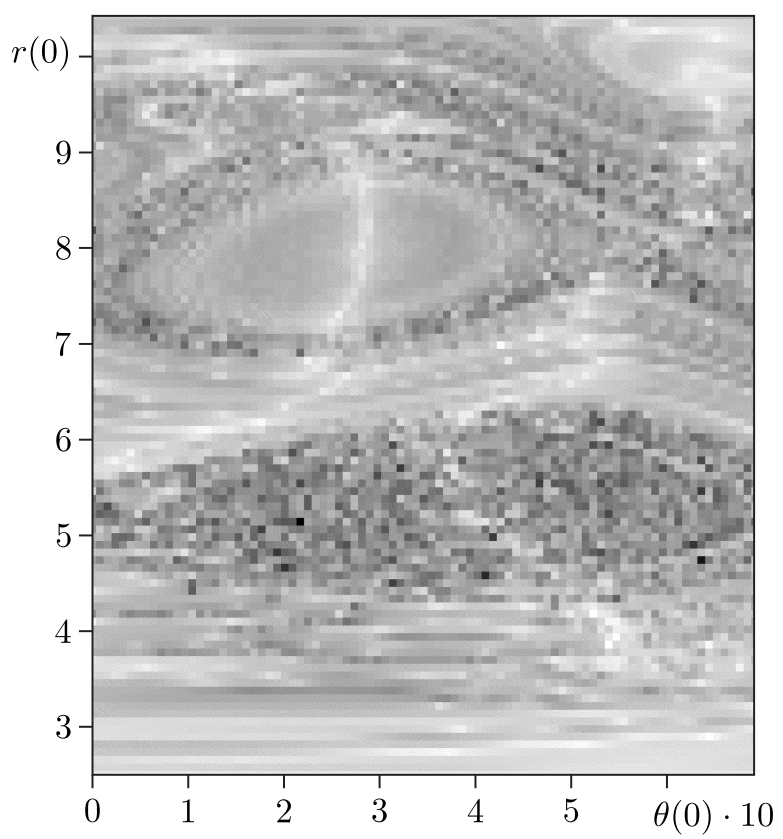

(a)

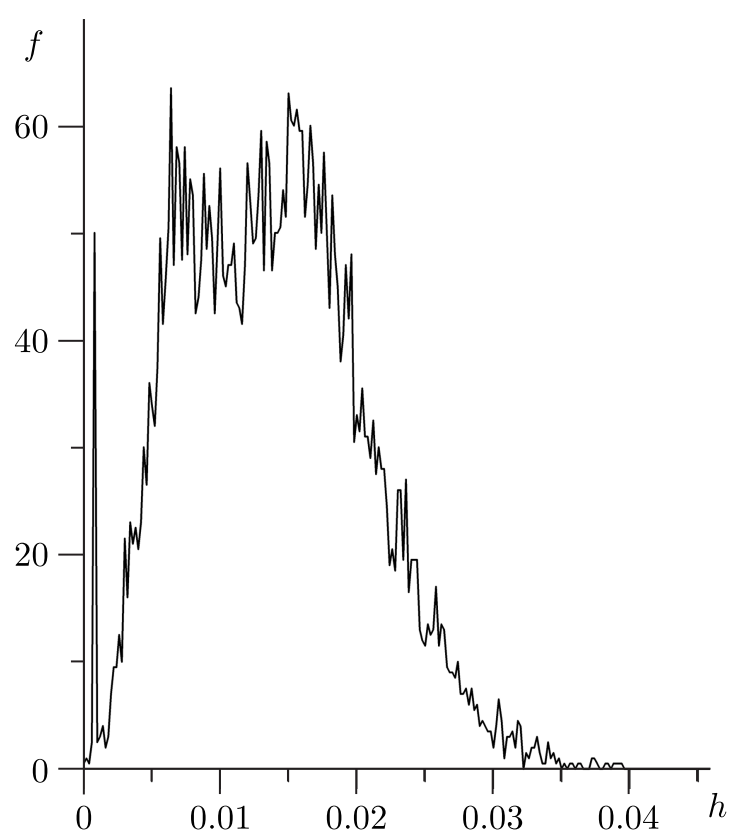

(b)

Fig. 5. (a) Inverse grayscale image of the finite-time Lyapunov exponent (FTLE) in the flow segment $0 \leqslant \theta(0) \lesssim 0.69$ (black indicates the highest exponent, and white, the lowest one); (b) FTLE distribution function $\left(\gamma=\gamma_{c}, t=1000 ; \theta(0)\right.$ and $r(0)$ are the initial polar coordinates of the tracer particles).

chain generation $\left(\gamma=\gamma_{c}\right)$. The images of the particles at both initial states obtained at $t=1000$ (i. e., for the upper limit of the power laws obtained above) are displayed in Fig. 7. Since the pseudoseparatrix contours are determined at $t=0$ in the comoving reference frame and remain fixed in time, the problem of spreading the particles from these initial states is actually solved. The location of the pseudoseparatrix contours shown in Fig. 7a allows the conclusion that the lateral transport of the particles takes place in the flow regions adjacent to the channel walls, whereas there is no lateral transport in the area between the chains. As can be seen from Fig. 7a, all particles leave the initial location in both chains, while in Fig. 7b a large number of particles remain inside the external chain. This is consistent with the location of the regions of Lagrangian chaos in Fig. 5a. At the same time, it was found that there is no cross-flow transport in the multiharmonic regimes (arising at $\gamma=\gamma_{a}$ and $\gamma=\gamma_{b}$ ). Although a large number of particles cross the pseudoseparatrix contours, they are localized in the narrow band adjoining the chain boundaries, which is confirmed by computing and plotting the particle orbits at $t \lesssim 1000$. The thickness of the bands does not change essentially in time and slightly increases when $\gamma$ passes from $\gamma_{a}$ to $\gamma_{b}$. Such a behavior can be explained by the phenomenon that was characterized in $[2,17]$ as a barrier to cross-jet transport.

To describe the lateral transport quantitatively, we placed $N_{01}=17700$ and $N_{02}=10660$ tracer particles inside the pseudoseparatrix contours of the external and internal chains, respectively, and the total number of particles located between the fixed circles $r=r_{1,2}$ and the channel walls in Fig. 7b were determined. The temporal dependences of the related number of particles $n_{1,2}=N_{1,2} / N_{01,2}$, where $N_{1,2}$ is the total number of particles in the regions $r_{1} \leqslant r<r_{0}+b$ and $r_{0}-b \leqslant r<r_{2}$ adjoining the external and internal chains, respectively, were computed separately in the chaotic regime $\left(\gamma=\gamma_{c}\right)$. The results of computations for $r_{1}=9.068$ and $r_{2}=4.654$ 


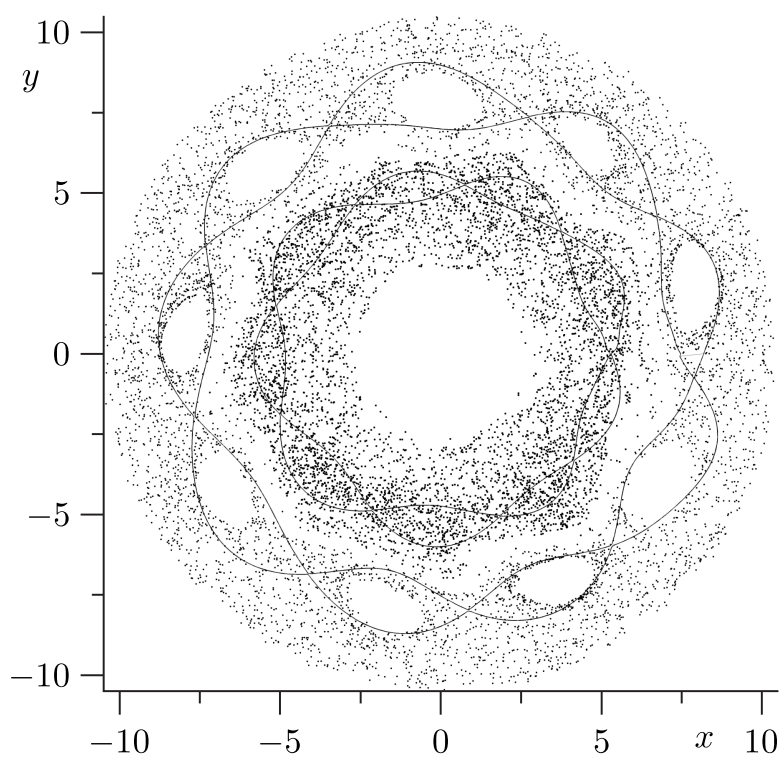

(a)

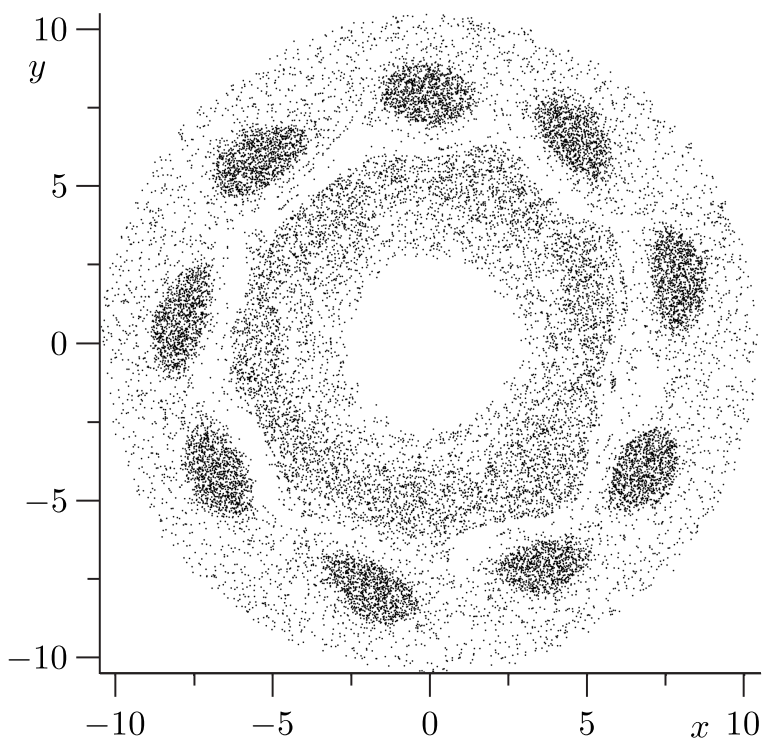

(b)

Fig. 6. Plots of tracer particles in the annular jet at $t=1000$ for $\gamma=\gamma_{c}$ : (a) 6000 particles in each chain initially distributed along the pseudoseparatrix contours shown by the solid curves, and (b) 13600 and 7800 particles initially localized inside the external and internal pseudoseparatrix contours, respectively. The plots are drawn in the frame of reference rotating with the angular frequency of the external chain $\Omega=0.0016$.

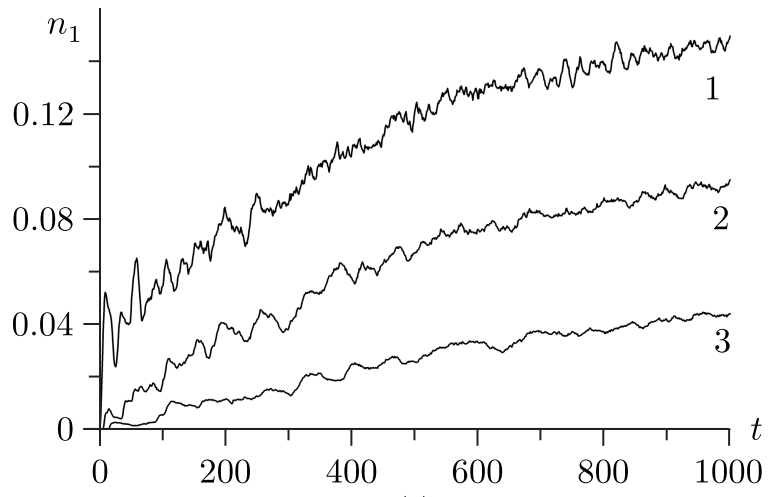

(a)

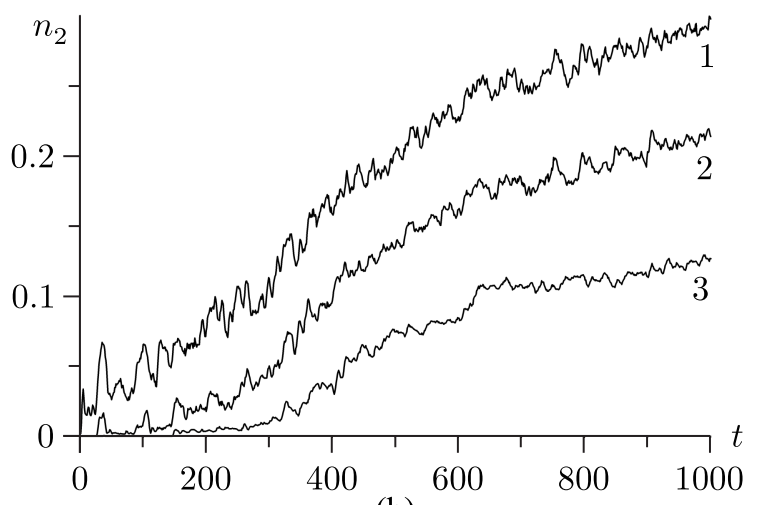

(b)

Fig. 7. Temporal dependence of the relative number of tracer particles $n_{1,2}$ located in the regions $r_{1} \leqslant r<10.5$ and $2.5 \leqslant r<r_{2}$ for the initial conditions specified inside the pseudoseparatrix contours shown in Fig. 7a: (a) $r_{1} \leqslant r<10.5$, curves 1, 2 and 3 correspond to $r_{1}=9.068,9.545$ and 10.02, respectively, and (b) $2.5 \leqslant r<r_{2}$, curves 1,2 and 3 correspond to $r_{2}=4.654,4.295$ and 3.936 , respectively.

corresponding to the maximal deviations of the pseudoseparatrix contours and for the values of $r_{1}$, and $r_{2}$ beyond these deviations are presented in Fig. 8. The unsteadiness of the chains, which is clearly visible in the images in Fig. 3 and is confirmed by the spectra in Fig. 6, gives rise to alternative crossing of the circles $r=r_{1,2}$ by tracer particles, thereby fairly small oscillations of $n_{1,2}$ occur. The increase of $n_{1,2}$ in time means that there exist lateral fluxes of fluid particles from the chains. Curves 1 in Figs. $8 \mathrm{a}$ and $8 \mathrm{~b}$, corresponding to the maximal deviation of the 
pseudoseparatrix contours, demonstrate a jump-like increase of $n_{1,2}$ at $t>0$. Really, there exists a small time interval (of about 20) over which $n_{1,2}$ increase from zero to the initial values displayed by curves 1 . Therefore, these jumps can be explained by the contribution of the particles stopped by the flow barrier to lateral transport. When the boundaries $r=r_{1,2}$ are shifted from the circles of maximal deviation, the jumps decrease and turn to zero, which is demonstrated by curves 2 in Figs. 8a and 8b. Finally, if the boundaries are shifted greatly enough, there are no particles in a detached area in a certain time interval, as is shown by curves 3 in Figs. $8 \mathrm{a}$ and $8 \mathrm{~b}$. This is the case of "pure" lateral transport that is not limited by the flow barriers.

Figure 8 shows that the cross-flow transport characterized by the magnitude of $n_{1,2}$ is stronger for the internal chain, which can be explained by a wider area of Lagrangian chaos in Fig. 5a. At the same time, there is no cross-flow transport in the case of multiharmonic regimes, when the flow barriers provide localization of tracer particles near the chains $\left(n_{1,2}\right.$ become zero at sufficiently large shifting of the boundaries $\left.r=r_{1,2}\right)$. The reason is that only in the chaotic regime of Eulerian fields the size of the regions of Lagrangian chaos becomes large enough for both the internal and the external chains, as can be seen in Fig. 5a. Thus, the analysis has shown that, in the chaotic regime of structure chain generation, the passive scalar is able to pass through the flow barrier, thereby the cross-flow chaotic transport occurs.

\section{References}

[1] Aref, H., Blake, J.R., Budišić, M., Cardoso, S.S.S., Cartwright, J.H.E., Clercx, H. J.H., El Omari, K., Feudel, U., Golestanian, R., Gouillart, E., van Heijst, G. J. F., Krasnopolskaya, T. S., Le Guer, Y., MacKay, R. S., Meleshko, V.V., Metcalfe, G., Mezić, I., de Moura, A.P. S., Piro, O., Speetjens, M.F.M., Sturman, R., Thiffeault, J.-L., and Tuval, I., Frontiers of Chaotic Advection, Rev. Modern Phys., 2017, vol. 89, no. 2, 025007, 66 p.

[2] Branicki, M. and Wiggins, S., Finite-Time Lagrangian Transport Analysis: Stable and Unstable Manifolds of Hyperbolic Trajectories and Finite-Time Lyapunov Exponents, Nonlinear Proc. Geophys., 2010, vol. 17, no. 1, pp.1-36.

[3] Chkhetiani, O. G., Kalashnik, M. V., and Chagelishvili, G.D., Dynamics and Blocking of Rossby Waves in Quasi-Two-Dimensional Shear Flows, JETP Lett., 2015, vol. 101, no. 2, pp. 79-84; see also: Pis'ma v Zh. Ėksper. Teoret. Fiz., 2015, vol. 101, no. 2, pp. 84-89.

[4] del-Castillo-Negrete, D., Asymmetric Transport and Non-Gaussian Statistics of Passive Scalars in Vortices in Shear, Phys. Fluids, 1998, vol. 10, no. 3, pp. 576-594.

[5] Dolzhanskii, F. V., Krymov, V.A., and Manin, D. Yu., Stability and Vortex Structures of QuasiTwo-Dimensional Shear Flows, Sov. Phys. Usp., 1990, vol. 33, no. 7, pp. 495-520; see also: Uspekhi Fiz. Nauk, 1990, vol. 160, no. 7, pp.1-47.

[6] Finn, J.M. and del-Castillo-Negrete, D., Lagrangian Chaos and Eulerian Chaos in Shear Flow Dynamics, Chaos, 2001, vol. 11, no. 4, pp. 816-832.

[7] Haines, K. H. and Malanotte-Rizzoli, P., Isolated Anomalies in Westerly Jet Streams: A Unified Approach, J. Atmos. Sci., 1991, vol. 48, no. 4, pp. 510-526.

[8] Koshel, K. V. and Prants, S. V., Chaotic Advection in the Ocean, Physics-Uspekhi, 2006, vol.49, no. 11, pp. 1151-1178; see also: Uspekhi Fiz. Nauk, 2006, vol.176, no. 11, pp. 1177-1206.

[9] Liu, Y., Wilson, C., Green, M. A., and Hughes, C. W. J., Gulf Stream Transport and Mixing Processes via Coherent Structure Dynamics, J. Geophys. Res. Oceans, 2018, vol.123, no. 4, pp. 30143037.

[10] Reutov, V.P. and Rybushkina, G. V., Anomalous Transport of a Passive Scalar at the Transition to Dynamical Chaos in a Barotropic Shear Layer, Eur. J. Mech. B Fluids, 2019, vol. 74, pp. 211-218. 
[11] Reutov, V.P. and Rybushkina, G. V., Dynamical Model for the Anomalous Transport of a Passive Scalar in a Reverse Barotropic Jet Flow, Russian J. Nonlinear Dyn., 2019, vol. 15, no. 3, pp. 251-260.

[12] Reutov, V.P. and Rybushkina, G. V., Transition to the Dynamical Chaos and Anomalous Transport of a Passive Scalar in the Annular Kolmogorov Flow, Phys. Fluids, 2020, vol. 32, no. 10, 106601, 10 p.

[13] Rogerson, A. M., Miller, P.D., Pratt, L.J., and Jones, C. K.R. T., Lagrangian Motion and Fluid Exchange in a Barotropic Meandering Jet, J. Phys. Oceanogr., 1999, vol. 29, no. 10, pp. 2635-2655.

[14] Ryzhov, E. A. and Koshel, K. V., Advection of Passive Scalars Induced by a Bay-Trapped Nonstationary Vortex, Ocean Dynamics, 2018, vol.68, pp. 411-422.

[15] Samelson, R. M., Fluid Exchange across a Meandering Jet, J. Phys. Oceanogr., 1992, vol. 22, no. 4, pp. 431-440.

[16] van de Konijnenberg, J. A., Nielsen, A. H., Rasmussen, J. J., and Stenum, B., Shear-Flow Instability in a Rotating Fluid, J. Fluid Mech., 1999, vol. 387, pp. 177-204.

[17] Wiggins, S., The Dynamical Systems Approach to Lagrangian Transport in Oceanic Flows, Annu. Rev. Fluid Mech., 2005, vol.37, pp. 295-328. 